\title{
Pineal Region Tumors
}

\author{
Michael C. Boyd and Paul Steinbok
}

\begin{abstract}
The management of pineal region tumors has changed considerably since the introduction of microsurgery and CT scanning. Analysis of our own series of 36 patients, comparing those treated in the pre-CT scan and microsurgery era with those treated afterwards, shows the important role these two techniques play. Current controversies in treatment and our own approach to management of pineal region tumors are discussed.
\end{abstract}

RÉSUMÉ: Tumeurs de la région de la pinéale La conduite à suivre pour les tumeurs de la région pinéale a considérablement changé depuis l'introduction de la microchirurgie et de la tomodensitométrie. Dans notre propre série de 36 patients la comparaison des résultats pré et post l'introduction de ces techniques démontre leur importance. Nous discutons aussi de certaines controverses thérapeutiques et présentons notre propre approche.

Can. J. Neurol. Sci. 1985; 12: 35-38

During the last decade, the approach to management of pineal region tumors has changed significantly as a result of computer tomographic (CT) scanning, microsurgery and clarification of the pathological classification of these lesions (Borit, 1981). The $25 \%$ to $70 \%$ operative mortality as reported by Schmideck in 1977 is no longer acceptable, as recently several authors have had excellent results with surgery (Handa and Yamashita, 1981; Neuwelt et al, 1979; Raimondi and Tomita, 1982; Reid and Clark, 1978; Sano, 1982). In addition, CT scanning allows not only a presumptive preoperative diagnosis but, combined with CSF tumor markers, permits a close follow-up of treatment (Chang et al, , 1981; Jooma and Kendall, 1983; Neuwelt et al., 1979). Finally, knowledge of the exact pathological diagnosis can insure that the appropriate therapy is instituted (Borit, 1981).

We have reviewed the experience with pineal region tumors at the Vancouver General Hospital from 1950 to 1983. The methods and results of treatment prior to the availability of CT scanning and microsurgery are compared to those after these devices became readily accessible. A plan for the management of such cases is discussed.

\section{Materials}

The series includes 36 patients, 25 males and 11 females. The age distribution is shown in Figure 1. Of these, 16 presented prior to the use of CT scanning. The signs and symptoms at presentation are shown in Table 1 . Both operative resection and radiation were used as primary methods of treatment.
Excluding shunts, there were 30 intracranial operative procedures performed either for tumor biopsy or resection. Eighteen of these made use of an operating microscope. The various pathological diagnoses obtained are shown in Table 2 . Twentysix patients had radiation therapy at some time during their course of treatment.

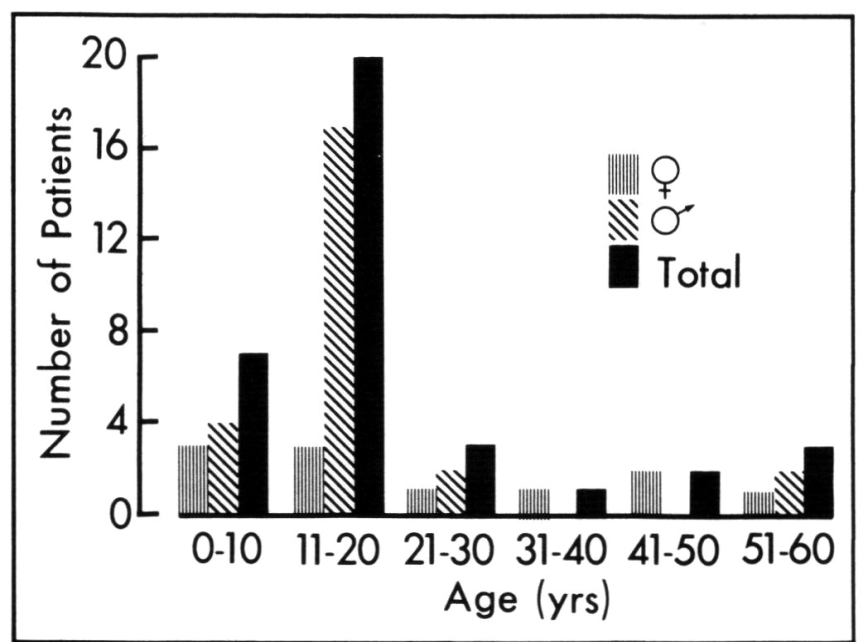

Figure I - Age Distribution of Patients with Pineal Region Tumours. 
Table 1: Presenting signs and symptoms in 36 patients with pineal region tumors

\begin{tabular}{lrlr}
\hline \multicolumn{1}{c}{ Symptoms } & & \multicolumn{1}{c}{ Signs } \\
\hline Headache & 26 & Parinaud's Syndrome & 20 \\
Nausea/Vomiting & 17 & Papilloedema & 17 \\
Diplopia & 12 & Truncal Ataxia & 11 \\
Lethargy & 5 & Hemiparesis & 8 \\
Personality Change & 3 & Visual Field Defects & 7 \\
Polyuria & 3 & Abducens Palsy & 4 \\
Decreased Memory & 2 & Facial Nerve Palsy & 2 \\
Dizziness & 2 & Macrocephaly & 2 \\
Tinnitus & 1 & Hypopituutarism & 2 \\
Hearing Loss & 1 & Cafe au Lait Spots & 2 \\
Photophobia & 1 & Cauda Equina Syndrome & 1 \\
\hline
\end{tabular}

Table 2: Pathological diagnosis with corresponding age and sex distribution of 36 patients with pineal region tumors

\begin{tabular}{lllll}
\hline \hline $\begin{array}{l}\text { Pathological } \\
\text { Diagnosis }\end{array}$ & Number & $\begin{array}{c}\text { Ages of } \\
\text { Males }\end{array}$ & $\begin{array}{c}\text { Ages of } \\
\text { Females }\end{array}$ \\
\hline Germinomas & 8 & $14,15,15,16,17,17,17$ & 33 \\
Astrocytomas & 4 & 4,13 & 5,15 \\
Benign Teratomas & 4 & $5,8,18,21$ & - \\
Pineocytoma & 3 & 3 mos., 17 & 57 \\
Arachnoid Cyst & 2 & 2 & 13 \\
Dermoid Cyst* & 1 & 22 & - \\
Pineoblastoma & 1 & 12 & - \\
Sarcoma & 1 & 10 & 15 \\
Oligodendroglioma & 1 & - & 46 \\
Cavernous & 1 & - & - \\
$\quad$ Hemangioma & 1 & 59 & - \\
Meningioma & 1 & 14 & $10,14,21,41$ \\
\hline Embryonal Cell Ca. & 9 & $13,16,19,29,51$ & \\
Undiagnosed & &
\end{tabular}

(*This Patient also had a germinoma, see text.)

\section{RESULTS}

Our series is similar to others with respect to age and sex distribution. In addition, the clinical presentations are comparable. The most common finding was evidence of increased intracranial pressure (32 patients) followed in decreasing frequency by Parinaud's syndrome (20 patients), ataxia (11 patients) and pyramidal tract abnormalities (10 patients). Ten patients had both increased ICP and Parinaud's syndrome, while 8 had increased ICP, Parinaud's syndrome and ataxia, and 3 had evidence of all four of the above signs and symptoms. These patterns of pineal region lesions are well established (Sano, 1982; Schmidek, 1977; Smith, 1974).

A surprisingly large number of patients (11) had symptoms for more than 12 months prior to diagnosis. With the exception of a single case, all of these presented prior to the advent of CT scanning. They generally had symptoms of increased intracranial pressure and, if investigated, frequently had evidence of hydrocephalus on air studies, angiograms or plain X-rays. A pineal region lesion was often suspected in the differential diagnosis. In several cases, however, the investigations had been delayed because the symptoms and signs were either not recognized or were not severe enough to warrant invasive studies. CT scanning now offers an effective, noninvasive means of evaluating the pineal region so that an early diagnosis may be made.

Since its introduction in 1968 , the operating microscope has been used in 18 of 22 intracranial procedures performed on these patients. Included were 17 infratentorial- supracerebellar approaches and one transparietal approach. In addition, 4 burr hole biopsies were done during this time. Using the microscope 6 biopsies and 4 partial resections were carried out. There were 8 cases in which the surgeon felt he had achieved a total removal of all tumor tissue. Eleven patients improved post-operatively, 7 remained unchanged and none deteriorated. No significant correlation could be made between severity of presenting symptoms and whether or not the patient improved. Although most patients presented with signs and symptoms of increased intracranial pressure and Parinaud's syndrome, several in both groups had further symptoms and signs of cerebellar or long tract dysfunction. Ten out of thirteen patients who had total or partial removal of their tumors improved. Two out of five biopsied patients improved, and in both of these arachnoidal cysts were drained. Total removal of the tumor was achieved with two teratomas, two arachnoidal cysts, and one each of pineocytoma, grade 1 astrocytoma, meningioma and dermoid tumor. Partial removal was done on two grade 1 astrocytomas, two germinomas and one meningioma. Biopsies were done on two arachnoid cysts, two pineocytomas and one grade 1 astrocytoma.

One patient, who had a total resection of a benign dermoid cyst, remained well for five and one half years at which time the tumor recurred. A burr hole biopsy showed a germinoma. Despite radiation therapy he died two and one half months after the second operation. Even in retrospect pathologic examination of the original tumor showed a dermoid cyst with no evidence of germinoma.

In 8 cases of pineal region tumors operated on prior to the use of an operating microscope, only one patient improved. Five were unchanged and two deteriorated. Both of the patients who deteriorated had three or more of the presenting signs and symptoms. One of them, who had a total resection of a benign teratoma through a suboccipital transtentorial approach, died two days after surgery. The single patient who improved had a total removal of a low grade glioma via a transcallosal approach. Of the remainder, five patients had biopsies including two germinomas, and one each of an astrocytoma grade 2, astrocytoma grade 3, and medulloblastoma. There was also one negative exploration. The patient with the medulloblastoma deteriorated, the remainder were unchanged. Three of these procedures were infratentorial-supracerebellar, three transcallosal-transventricular, one occipital-transtentorial and one transparietal.

The introduction of the microscope has resulted in an increased number of complete or partial resections. Prior to the availability of the microscope, resection was attempted in only $25 \%(2 / 8)$ of cases, while this figure increased to $67 \%(12 / 18)$ with its use. There were $6 / 18(33 \%)$ and $6 / 8(75 \%)$ biopsies done with and without the microscope respectively. With better vision afforded by the microscope, most tumors underwent at least a partial debulking, if not attempts at total removal.

A tissue diagnosis was established prior to radiation therapy in 17 of 26 patients. Of these 17, 7 improved (4 germinomas, 1 pineocytoma, 1 astrocytoma grade 1, 1 teratoma), 3 remained unchanged (1 pineocytoma, 1 astrocytoma grade 1, 1 germinoma), 
4 deteriorated ( 2 germinomas, 1 embryonal cell carcinoma, 1 meningioma), 2 died within 3 months of treatment ( 1 germinoma, 1 pineoblastoma) and 1 was lost to follow-up (anaplastic astrocytoma). In the 3 patients with germinomas who did poorly, two developed pan-hypopituitarism, and one was in poor general health prior to treatment due to a delay in diagnosis. Of the undiagnosed patients, 3 improved, 2 were unchanged, 3 deteriorated and 1 was lost to follow-up. A tissue diagnosis was subsequently obtained in 4 of these. One sarcoma and 1 cavernous hemangioma were found at autopsy 1 and 10 years later respectively. Biopsies from two other patients showed an astrocytoma grade 1 , and a benign teratoma 7 and 3 years respectively post-radiation therapy.

We have also measured the CSF levels of tumor markers and have done CSF cytology in some patients. The human chorionic gonadotrophin (HCG) beta-subunit was within normal limits in four patients. One of these had an astrocytoma grade 1 on biopsy, while two others were treated with radiation without a tissue diagnosis. The fourth patient, who was found to have a normal HCG level, had an elevated level of alpha-fetoprotein. He had presented with a cauda equina syndrome secondary to compression by an embryonal cell carcinoma. A CT scan of his head revealed a pineal region tumor. Presumably, the lumbar tumor was a metastasis. Five other patients had CSF alphafetoprotein levels assessed, and all were within normal limits. One patient had a benign teratoma, one an astrocytoma grade 1 and three had local radiation therapy without tissue diagnosis. Of the three CSF cytological assessments done, one showed cells diagnostic of a germinoma. The remaining two studies were not helpful in obtaining a tissue diagnosis.

Follow-up of these patients shows results comparable to that found in the literature for germinomas. Of the 7 with biopsy proven germinomas, all received radiation therapy. One died within 3 months of treatment (described above) and two died at 2 and 8 years of unrelated causes. Of the remaining, three were well after more than 5 years and one after 2 years follow-up. The number of other tumors in this series have been too small to permit any comparative conclusions to be drawn.

\section{Discussion}

The characteristics of benign and malignant pineal region tumors have been well documented in the literature (Sano, 1982; Schmidek, 1977; Smith and Estridge, 1974). Once a tissue diagnosis is obtained, there appears to be little disagreement over treatment for particular tumor types. In general, radiosensitive tumors, such as germinomas, should be treated primarily by radiation therapy and surgically debulked only if very large. Less radiosensitive tumors, including astrocytomas, pineoblastomas, pineocytomas and other germ cell tumors (embryonal cell carcinoma and malignant teratoma), should have as much surgical debulking as possible as well as radiation therapy. All benign symptomatic tumors should have a total resection if possible (Handa and Yamashita, 1981; Neuwelt et al., 1979).

Radiation therapy without doubt plays an important role in the treatment of pineal region tumors (Griffin et al., 1981). However, whether or not it should be used blindly without a tissue diagnosis is questionable (Neuwelt, 1984). We agree with those authors who suggest that patients suspected of having germinomas may undergo a trial of radiation therapy (Handa and Yamashita, 1981; Jooma and Kendall, 1983; Griffin et al.,
1981) without tissue diagnosis. If this is ineffective in dissolving the tumor after 2000 rads in 2 weeks, then a biopsy should be obtained as the diagnosis of a germinoma is suspect. All other lesions should have a tissue diagnosis prior to radiation.

Obtaining a tissue diagnosis is not a simple procedure. The pineal region is deep within the brain and until recently has been relatively inaccessible. With the operating microscope and better anesthetic techniques, the results of surgery in this area have improved. Our results indicate that surgeons are more willing to attempt not only biopsies but extensive resections with the microscope. Although the numbers are small there appears to be an improvement in outcome also.

A tissue diagnosis may be obtained by stereotactic biopsy through a burr hole or by a direct surgical approach using the operating microscope. We have had success with the supracerebellar-infratentorial approach to the pineal region and now use it almost exclusively. In our hands the procedure has resulted in minimal morbidity and no mortality. However, such an operation is a major undertaking and we prefer not to operate at all if a presumptive tissue diagnosis can be made by less invasive means.

CT scanning has made it possible to follow the treatment of these patients closely. It is especially beneficial when no biopsy has been obtained prior to beginning radiation therapy. In addition, CT scanning may aid in the early recognition of these lesions. In some cases, the pathological diagnosis may even be suggested. The typical CT scan appearance of a germinoma is an isodense to hyperdense mass which enhances homogeneously. The presence of cysts or inhomogeneity on enhancement should lead one to suspect a diagnosis other than a germinoma. Such information may further assist in treatment planning (Chang et al., 1981; Handa and Yamashita, 1981; Jooma and Kendall et al., 1983).

Although we have not used them extensively, others have found CSF tumor markers to be helpful in both diagnosis and follow-up (Neuwelt et al., 1979; Sano, 1982). If the CSF contains significantly elevated levels of HCG beta-subunit or alphafetoprotein, a presumptive diagnosis of choriocarcinoma, embryonal cell carcinoma or endodermal sinus tumor can be made. Likewise CSF cytology may also aid in diagnosis and subsequent treatment.

The most common tumor in our series, as in others (Jooma and Kendall, 1983; Sano, 1982) was a germinoma. Typically these occur in males and become symptomatic during the second decade of life. Rarely do they appear in males outside this age group or in females of any age. Should a pineal region lesion be found in a male younger than 10 or older than 20 years of age, or in a female of any age, a diagnosis other than a germinoma should be strongly suspected. This is particularly true if the CT scan does not have the characteristic features described above or if the level of CSF tumor markers is elevated.

With the above features in mind we now pursue the following course of investigation and treatment for pineal region tumors. Once the diagnosis of a pineal region tumor is suspected, radiological examinations, including CT scanning and sometimes angiography, and CSF markers and cytology are evaluated in an attempt to make a diagnosis without surgical intervention. If a presumptive diagnosis can be made with CSF analysis, appropriate treatment is begun. On the other hand, when CSF examination is negative, a trial of local radiation therapy to the tumor site is carried out if the radiological and epidemiological fea- 
tures suggest a germinoma: by that, we mean that the tumor on CT scan should be a homogeneously enhancing mass and the patient should be a male in the second decade of life. If these criteria are not fulfilled, surgical exploration is recommended. In the patients who are given a trial of radiation therapy, a CT scan is repeated after a total dose of 2,000 rads in two weeks. If the tumor has disappeared on this repeat CT scan, a presumed diagnosis of a germinoma is made and a full course of craniospinal radiation is completed. However, if there is no response or only partial response to the two week trial of radiation therapy, the diagnosis of germinoma is likely incorrect and surgical exploration for biopsy or resection is required.

If this protocol is followed, a number of patients with a germinoma will have appropriate treatment without surgery, and all patients with non-germinomatous tumors will have appropriate surgical exploration. It is important that one accepts only complete disappearance of the tumor on the post $2000 \mathrm{rad}$ CT scan as evidence of a germinoma. We have had one patient, a fifteen year old male, whose tumor changed little after 2000 rads. Instead of immediate exploration as required by our protocol, a further 3000 rads were delivered to the tumor area. The tumor developed a cystic appearance which gradually grew over three years with a concomitant deterioration in neurological function. He was finally explored. A benign teratoma was found and completely removed.

\section{REFERENCES}

Borit A (1981) History of tumors of the pineal region. Am. J. Surg. Path. 5: 613-620.

Chang CG, Kogeyama N, Kobayashi T, et al (1981) Pineal tumors; Clinical diagnosis, with special emphasis on the significance of pineal calcification. J. Neurosurg. 8: 656-667.

Giovannelli $G$ (1982) Pineal region tumors endocrinological aspects. Child's Brain, 9: 267-273.

Griffin BR, Griffin TW, Ton Dyk, et al (1981) Pineal region tumors: Results of radiation therapy and indications for elective spinal irradiation. J. Rad. Onc. Biol. Phys. 17: 605-608.

Handa $H$, Yamashita $\mathbf{J}$ (1981) Current treatment of pineal tumors. Neur. Med. Chir. 21: 147-148.

Jooma R, Kendall BE (1983) Diagnosis and management of pineal tumors. J. Neurosurg. 58: 654-665.

Neuwelt EA, Glasberg M, Frenkel E, et al (1979) Malignant pineal region tumors: A clinico-pathological study. J. Neurosurg. 51: 597-607.

Neuwelt EA (1984) Malignant pineal tumors: An update presented at the American Association of Neurosurgery Annual Meeting, San Francisco.

Raimondi A, Tomita T (1982) Pineal tumors in childhood: Epidemiology, pathophysiology and surgical approaches. Child's Brain, 9: 239-266.

Reid WS, Clark WK (1978) Comparison of infratentorial and transtentorial approaches to the pineal region. Neurosurgery, 3: 1-8.

Sano K (1982) Pineal region tumors: Problems in pathology and treatment. Clin. Neurosurg. 30: 59-91.

Schmidek HH (1977) Pineal Tumors. New York, Masson Pub. Inc.

Smith RA, Estridge MN (1974) Pineal Tumors. In: Handbook of Neurology, North-Holland Publishing Co., Amsterdam, Ch. 17, 648-665. 